\title{
Influence of PM Coating on PM Magnetization State Estimation Methods Based on Magnetoresistive Effect
}

\author{
Daniel Fernandez ${ }^{1}$, David Reigosa ${ }^{1}$, Juan Manuel Guerrero ${ }^{1}$, Z. Q. Zhu ${ }^{2}$, Fernando Briz ${ }^{1}$ \\ ${ }^{1}$ University of Oviedo. Dept. of Elect., Computer \& System \\ Engineering, Gijón, 33204, Spain. \\ ${ }^{2}$ University of Sheffield. Department of Electronic and \\ Electrical Engineering, UK. \\ fernandezalodaniel@uniovi.es, diazdavid@uniovi.es, guerrero@uniovi.es, z.q.zhu@sheffield.ac.uk, fernando@isa.uniovi.es
}

\begin{abstract}
Variable flux PMSMs (VF-PMSMs) are gaining importance, especially in automotive applications. The use of such machines will require the development of methods to Destimate the permanent magnet (PM) magnetization state. PM magnetization state estimation methods reported in literature include BEMF and high frequency signal injection. One advantage of high frequency signal injection methods is that they can operate over the whole speed range, with practically no interference with the regular operation of the machine. Magnetization estimation using high frequency injection relies on the variation of the $d$-axis high frequency inductance with the saturation produced by the magnets. Alternatively, the changes of the magnet electrical resistance due to the magnetoresistive effect can also be used for this purpose. These methods have been tested with different materials, including NdFeB, AlNiCo and SmCo magnets. NdFeB magnets are usually protected from oxidation using $\mathrm{NiCuNi}$ coating. However, NiCuNi also shows magnetoresistance effect, and can affect therefore the performance of the method when used with magnets having NiCuNi coating. This paper studies the effects of PM coating on the performance of methods which estimate the magnetization state of the magnet using the magnetoresistance effect by means of high frequency signal injection. The analysis will include NdFeB, AINiCo and SmCo magnets. ${ }^{1}$
\end{abstract}

Index Terms - High frequency signal injection, Magnetization State, Magnetoresistance, $\mathrm{NiCuNi}$ coating,

\section{NOMENCLATURE}

\begin{tabular}{|c|c|}
\hline$i_{h f p}^{p}$ & Coil high frequency current referred to primary side \\
\hline$v_{h f p}^{p}$ & Coil high frequency voltage referred to primary side \\
\hline$\omega_{h f}$ & High frequency signal angular speed \\
\hline$R_{h f p}$ & Overall high frequency resistance \\
\hline$L_{h f p}$ & Overall high frequency impedance \\
\hline$R_{h f p}^{p}$ & Coil high frequency resistance referenced to primary side \\
\hline$R_{h f s}^{p}$ & PM high frequency resistance referred to primary side \\
\hline $\mathrm{R}_{h f F E p}^{p}$ & $\begin{array}{l}\text { Core high frequency resistance referred to the primary } \\
\text { side }\end{array}$ \\
\hline$M R$ & Magnetoresistance coefficient of the magnet \\
\hline$\rho$ & Resistivity of the material (magnet?) \\
\hline$\beta$ & $\begin{array}{l}\text { Sensitivity of the material (magnet?) resistivity to an } \\
\text { external field }\end{array}$ \\
\hline
\end{tabular}

${ }^{1}$ This work was supported in part by Spanish Ministry of Education, Culture and Sports through "José Castillejo Program" under grant PX15/00354, by Spanish Ministry of Economy and Competitiveness under the project MINECO-17-ENE2016-80047-R and by Regional Ministry of Education, Culture and Sport of the Principality of Asturias through "Severo Ochoa Program" under Grant BP-13067.

\section{INTRODUCTION}

MSMs have been widely studied and used during last decade due to their high efficiency, high power density and good dynamic response. The increased cost of rare earth materials during the last years has increased the interest in machine designs using less magnet, as well as on the substitution of rare earth by cheaper materials, e.g. AlNiCo, Ferrite, etc. [1]-[5]. However, these substitutes raise other concerns, as they can be demagnetized relatively easily, e.g. due to temperature increases or even to the current injected in the stator windings under normal operation [6]-[8].

Easiness of demagnetization can be advantageous for certain machine designs, e.g. VF-PMSMs. VF-PMSMs can use either low coercivity magnets, or combine high coercivity magnets (i.e. $\mathrm{NdFeB}$ ) and low coercivity magnets (e.g. Ferrite, AlNiCo...) [9]-[11].

VF-PMSMs magnetization state can be changed during normal operation of the machine by applying a current pulse to the stator terminals [10]. Knowledge of the magnetization state after a magnetization/demagnetization process is critical. Direct magnetization state measurement can be done by inserting a magnetometer in the machine airgap or by installing field sensors under the magnet [12]. However, both methods imply changes in the machine design as well as additional sensors, cabling and electronics, which eventually adversely impact system robustness and cost.

The unfeasibility of direct measurement has boosted the interest in magnetization state estimation methods [13]-[15]. Magnetization state estimation methods can be divided into Back-EMF and high frequency signal injection based methods. Back-EMF based methods estimate the PM magnetization state from the PM flux linkage, which is obtained from the machine terminal voltages and currents [16], without interfering at all with the normal operation of the machine. These methods can

TABLE I. NDFEB MAGNET SURFACE TREATMENTS

\begin{tabular}{|l|c|c|c|}
\hline & Aluminum & Nickel Plating & Epoxy Coating \\
\hline $\begin{array}{l}\text { Standard Thickness } \\
(\mu \mathrm{m})\end{array}$ & $5 \sim 20$ & $5 \sim 25$ & $10 \sim 30$ \\
\hline $\begin{array}{l}\text { Thermal humidity } \\
\text { resistance }\end{array}$ & Good & Excellent & Good \\
\hline Salt resistance & Poor & Good & Excellent \\
\hline Adhesive endurance & Excellent & Poor & Poor \\
\hline Insulation & Poor & Poor & Excellent \\
\hline *Source [18]
\end{tabular}


be used in the mid-to-high speed region, but are not viable at very low/zero speed due to the reduced magnitude of the Back-EMF. In addition, previous knowledge of some machine parameters (i.e. stator resistance, inductance maps...) is needed when operating under load conditions. High frequency signal injection based methods can estimate the magnetization state either from the $d$-axis high frequency inductance [14], which changes with the saturation level, or from the stator reflected magnet high frequency resistance [13],[14], which changes with the PM magnetization state due to the magnetoresistive effect [13]. Magnetoresistance based methods have been tested with $\mathrm{NdFeB}$, Ferrite and $\mathrm{SmCo}$ magnets, $\mathrm{NdFeB}$ showing the highest sensitivity, SmCo and Ferrite showing very modest values [14].

Previous studies have shown that coating can improve the magnetoresistance sensitivity in the machines equipped with Ferrite, AlNiCo or SmCo magnets [20]-[21]. However, thorough study of this effect has not been addressed yet.

Coating of $\mathrm{NdFeB}$ magnets is a normal practice as otherwise they can easily oxide by air moisture, salts and some gases [17]. Table I shows typical surface treatments used for $\mathrm{NdFeB}$ protection [18]. Nickel plating and epoxy are the most extended surface treatments a relatively thin coating being enough for this purpose. Nickel plating is especially effective as it provides a hermetic seal. In addition, it is one of the cheapest coating methods [17].

This paper analyses the effects of the PM coating on its magnetoresistance sensitivity, and its further use for magnetization state estimation purposes [25]. An experimental setup especially designed for this purpose will be used. The paper is organized as follows: Magnetoresistance effect is briefly reviewed in section II. High frequency signal injection for PM magnetization state estimation is presented in section III. Finally, experimental results are provided in section IV, while conclusions are presented in Section V.

\section{MAgnetoresistance EFFECT IN THIN FILM LAYERS}

Magnetoresistance is defined in (1) as the change of the material electrical resistivity when an external magnetic field is applied [19],[20], where $M R$ is the magnetoresistance, i.e. variation of resistivity due to the external field with respect to the initial resistivity, $\rho(0)$ is the resistivity of the material in the absence of magnetic field and $\rho(\mathrm{H})$ is the resistivity of the material and $H$ the strength of the magnetic field being applied.

$$
M R=\frac{\Delta \rho(\mathrm{H})}{\rho(0)}=\frac{\rho(\mathrm{H})-\rho(0)}{\rho(0)}
$$

The variation produced by the magnetoresistive effect over the absolute material resistance is defined by (2) where $\beta$ is the coefficient that links the PM flux and the resistance variation (3).

$$
\begin{aligned}
& R(\mathrm{H})=R\left(\mathrm{H}_{0}\right)\left(1+\beta\left(\mathrm{H}-\mathrm{H}_{0}\right)\right) \\
& \beta=\frac{R(\mathrm{H})-R\left(\mathrm{H}_{0}\right)}{\left(\mathrm{H}-\mathrm{H}_{0}\right) R\left(\mathrm{H}_{0}\right)}
\end{aligned}
$$

$$
\begin{aligned}
& \delta_{H}=\frac{\Delta R}{R(0)}=\frac{R_{\uparrow \uparrow}-R_{\uparrow \downarrow}}{R_{\uparrow \downarrow}} \\
& \beta(\mathrm{H}, \uparrow \uparrow, \uparrow \downarrow)=\frac{R(\mathrm{H}, \uparrow \uparrow)-R\left(\mathrm{H}_{0}, \uparrow \downarrow\right)}{\Delta \mathrm{H} R\left(\mathrm{H}_{0}, \uparrow \downarrow\right)}
\end{aligned}
$$

Not all materials have the same sensitivity to the magnetoresistance effect. As already mentioned, $\mathrm{NdFeB}$ magnets have a higher sensitive compared to Ferrite and SmCo magnets [14]. In addition, certain designs, e.g. the layer stack arrangement shown in Fig. 1, can be used to increase the sensitivity to magnetoresistance effect in magnetoresistive sensors, the sensitivity increasing with the number of layers [20]. Giant magnetoresistance (GMR) is defined when the variation of the electrical resistance is higher than $10 \%$ [22].

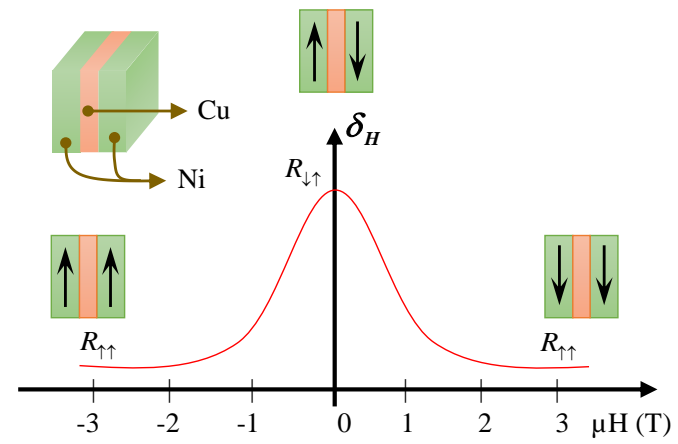

Fig.1 Magnetoresistance variations in multilayer structures.

a)

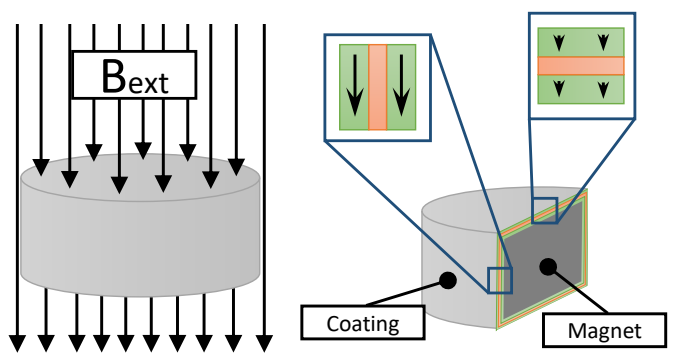

b)

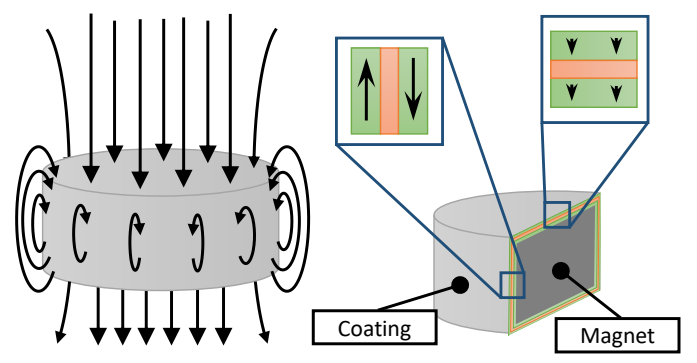

Fig.2 Magnetization direction of the coating layers when an external magnetic field is applied a) to a demagnetized magnet and b) to a magnetized magnet without external field.

A common arrangement to achieve GMR is a three-layer stack made of two ferromagnetic materials (e.g. Nickel, Iron...) and an electric conductor, non-ferromagnetic material (e.g. copper, gold..., see Fig. 1). The resistor model for GMRs is defined by (4) where $R_{\uparrow \downarrow}$ is the resistance with opposite magnetization in layers (i.e. in absence of $\underline{H}$ ), $R_{\uparrow \uparrow}$ is the 
resistance measured when the same direction of field $H$ is applied to the layers and $\delta_{H}$ is the GMR [19]. Therefore $\beta$ is function of both MR and $\delta_{H}$ (5).

It is interesting to note the similarities between the arrangements shown in Fig. 1, used to maximize the magnetoresistive effect in magnetoresistive sensors [23], [24] and the $\mathrm{NiCuNi}$ coating, commonly used with $\mathrm{NdFeB}$ magnets to avoid oxidation (see Fig. 2) [17]. Magnetic multilayer thinfilm structures are extremely sensitive magnetic field sensors as their electrical resistance change with the magnetization direction of their ferromagnetic layers [23]. Electrical resistance of PMs protected with NiCuNi coating is also highly sensitive to the magnetization direction of their ferromagnetic layers. Reduced values of the resistance occur when the magnet is fully demagnetized (Fig. 2a), while increased values occur when the magnet is fully magnetized due to flux leakage (Fig. 2b).

\section{MAGNet High FREQUENCY RESISTANCE MEASUREMENT}

The procedure used to measure the magnet high frequency resistance is presented in this section. The experimental setup that will be used is shown in Fig. 3. It consists of a magnetic core made of iron powder [13], [14] and a coil. The core was constructed using Fe-Si alloy iron powder blocks (BK8320-26 and CK2020-26, $\mu_{r}=26$ ) [13], [14]. The core has a cylindrical shape central column, the magnet having the same diameter as the central column [13], [14].
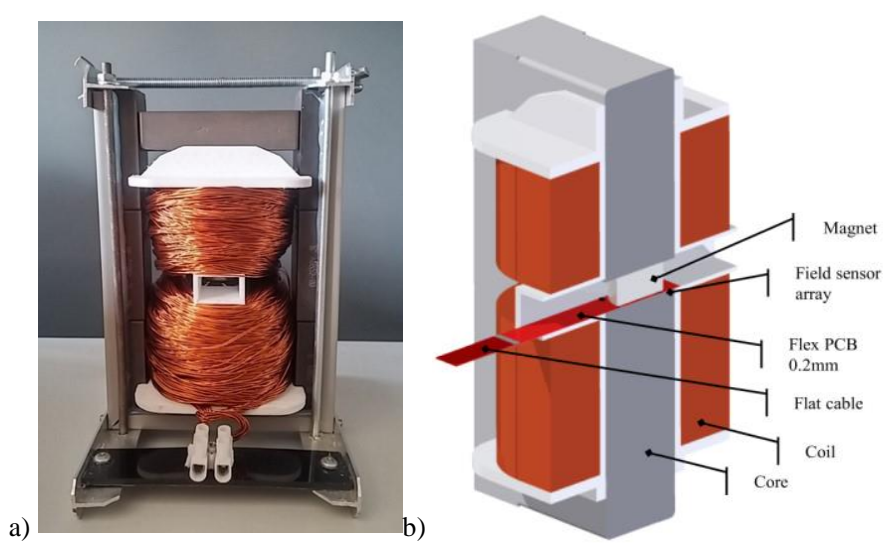

Fig. 3. Experimental setup used for PM magnetoresistance evaluation, (a) and section of the experimental setup, (b).

The coil in Fig. 3 is fed from an H-bridge using IGBTs (Fig. 4). The DC bus voltage is $\mathrm{Xx}, \mathrm{V}$, which is provided by a diode rectifier. Unipolar PWM I used to control the H-bridge to reduce to harmonic content of the current. A current control allows simultaneous injection of both DC current used to change the PM sample magnetization state and the high frequency current used to estimate the magnet sample magnetization state. The system parameters are shown in Table II. Fig. 5 shows the power converter control block diagram. A proportional integral regulator ("PI reg." in Fig. 5) controls the injected DC current $i_{D C p}^{p^{*}}$, while a resonant PI controller ("Resonant PI" in Fig. 5) controls the injected high frequency current $i_{h f p}^{p^{*}}$. A band-stop filter (BSF1 in Fig. 5) prevents the PI controller reaction to the high frequency current.

a)

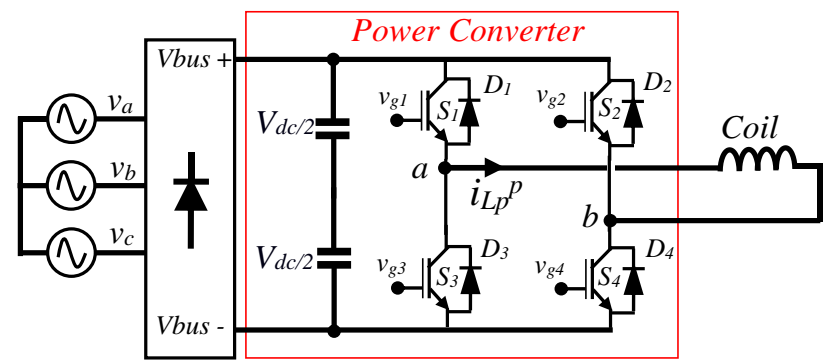

b)

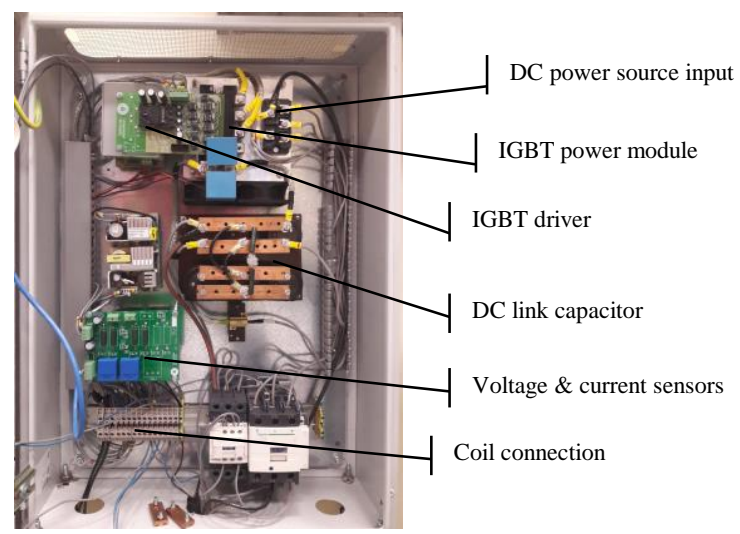

Fig. 4. a) H-Bridge power converter schematic representation and b) picture of the experimental setup.

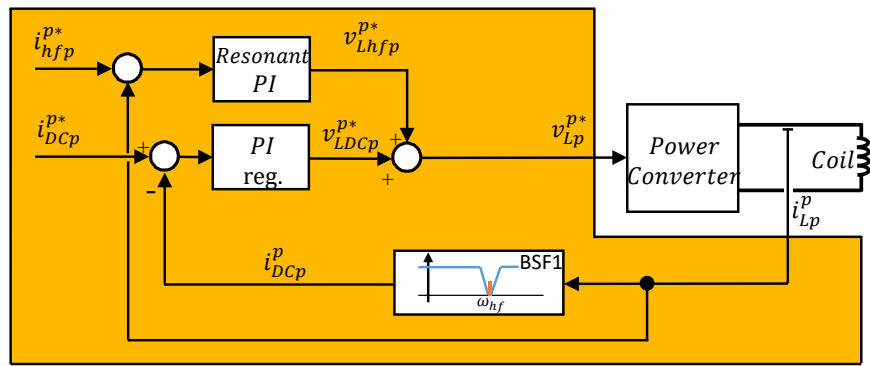

Fig.5. Block diagram of the DC and high frequency signal.

When the coil is fed with a high frequency current $i_{h f p}^{p}(6)$, the overall high frequency impedance is given by (7), where $v_{h f p}^{p}$ is the high frequency voltage, $Z_{h f p}$ is the overall high frequency impedance, while $R_{h f p}$ and $L_{h f p}$ are the overall high frequency resistance and inductance respectively. The real part of the high frequency impedance, $R_{h f p}$, is a function of the coil $R_{h f p}^{p}$, core $R_{h f F E p}^{p}$ and magnet $R_{h f s}^{p}$ high frequency resistance.

$$
\begin{aligned}
& i_{h f p}^{p}=I_{h f} \sin \left(\omega_{h f} \mathrm{t}\right) \\
& Z_{h f p}=\frac{v_{h f p}^{p}}{i_{h f p}^{p}}=\left(R_{h f p}+j \omega_{h f} L_{h f p}\right)=R_{h f}+j \omega_{h f} L_{h f} \\
& R_{h f F E p}^{p}=R_{h f p}-R_{h f p}^{p} \\
& R_{h f s}^{p}=\frac{R_{h f p}^{p} R_{h f F E p}^{p}-R_{h f p} R_{h f F E p}^{p}}{R_{h f p}-R_{h f p}^{p}-R_{h f F E p}^{p}}
\end{aligned}
$$


The reflected magnet high frequency resistance, $R_{h f s}^{p}$, can be estimated from the overall coil high frequency resistance, $R_{h f p}$. However, this requires decoupling of the coil ( $R_{h f p}^{p}$ ) and core $\left(R_{h F F E p}^{p}\right)$ contributions to the overall high frequency resistance.

\begin{tabular}{|c|c|c|c|c|c|}
\hline \multicolumn{2}{|c|}{ Coil Parameters } & \multicolumn{2}{|c|}{ Core Parameters } & \multicolumn{2}{|c|}{$\begin{array}{c}\text { Single Phase Inverter } \\
\text { Rated Parameters }\end{array}$} \\
\hline $\begin{array}{l}\text { Number of } \\
\text { turns }\end{array}$ & 490 & $\begin{array}{l}\text { Central column } \\
\text { diameter }(\mathrm{mm})\end{array}$ & 20 & $\begin{array}{l}\text { Switching } \\
\text { frequency }\end{array}$ & $10 \mathrm{kHz}$ \\
\hline $\begin{array}{c}\text { Resistance } \\
(\Omega)\end{array}$ & 0.699 & $\begin{array}{c}\text { Saturation, } B_{\text {sat }} \\
\text { (T) }\end{array}$ & 1.6 & Voltage & $380 \mathrm{~V}$ \\
\hline $\begin{array}{l}\text { Parallel } \\
\text { wires per } \\
\text { turn }\end{array}$ & 7 & $\begin{array}{c}\text { Relative } \\
\text { permeability, } \mu_{r}\end{array}$ & 26 & Current & $75 \mathrm{~A}$ \\
\hline $\begin{array}{l}\text { Inductance } \\
(\mathrm{mH})\end{array}$ & 12.5 & & & $B S F 1$ & $10 \mathrm{~Hz}$ \\
\hline$\alpha_{\mathrm{cu}}(1 / \mathrm{K})$ & $3.9 \mathrm{e}-3$ & & & $\begin{array}{c}\text { Bandwidth } \\
\text { of current } \\
\text { reg. }\end{array}$ & $200 \mathrm{~Hz}$ \\
\hline & & $v_{h f p}^{p}$ & & & \\
\hline
\end{tabular}

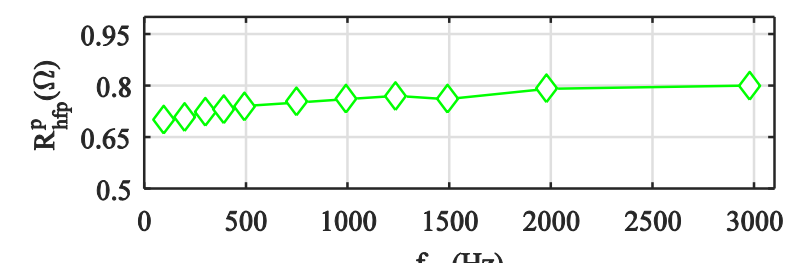

b)

$\mathrm{f}_{\mathrm{hf}}(\mathrm{Hz})$

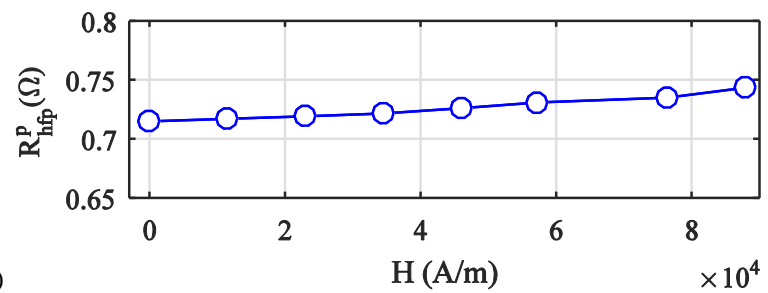

Fig. 6. a) Equivalent high frequency circuit of the experimental setup without core and magnet, b) coil high frequency resistance, $R_{h f p}^{p}$, for different frequencies, c) coil high frequency resistance, $R_{h f p}^{p}$, for different values of $\mathrm{H}$ values. $f_{h f}=250 \mathrm{~Hz}$ and $I_{h f}=1 \mathrm{~A}$.

The coil high frequency resistance $R_{h f p}^{p}$ can be approximated by removing the coil from the core and injecting a high frequency signal. The resulting equivalent high frequency circuit is shown in Fig. 6a. Fig. 6b shows $R_{h f p}^{p}$ vs. the frequency of the injected signal. Fig. $6 \mathrm{c}$ show $R_{h f p}^{p}$ vs. the strength of the magnetic field, $H$, which is produced by forcing a DC current into the coil. As expected, $R_{h f p}^{p}$ increases as the frequency does due to the skin effect (see Fig. 6b). Also as expected, $R_{h f p}^{p}$ slightly increases with $H$, meaning that the magnetoresistive effect in the coil (made of copper) cannot be negligible.
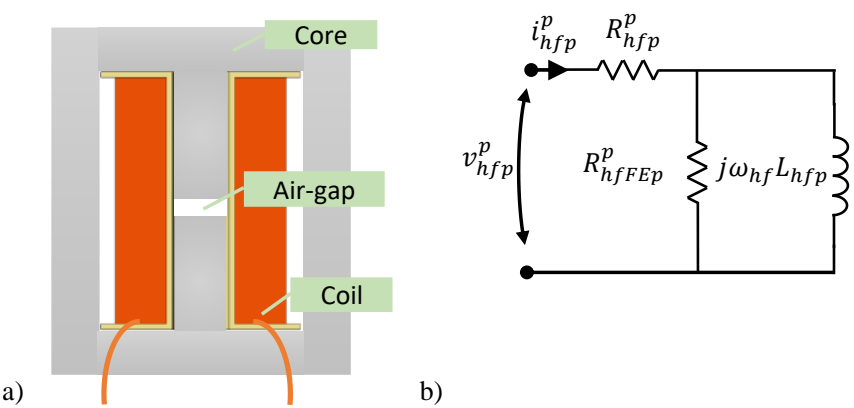

a)
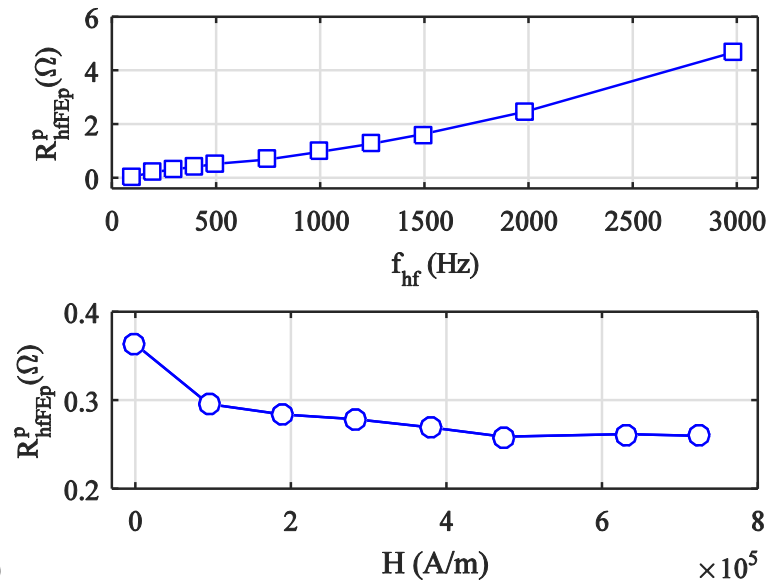

Fig. 7. a) Experimental setup configured for core high frequency resistance estimation (i.e. without magnet), b) equivalent high frequency circuit and c) core high frequency resistance vs. frequency of the injected, d) $R_{h f p}^{p}$ vs. H for $f_{h f}=250 \mathrm{~Hz}$ and $I_{h f}=1 \mathrm{~A}$.

The core high frequency resistance, i.e. $R_{h f F E p}^{p}$, can be estimated by injecting a high frequency signal into the coil with the coil and the core assembled, and replacing the magnet in the central column by a non-ferromagnetic material (see Fig. 7a). Fig. $7 b$ shows the equivalent circuit in this case, the core high frequency resistance being obtained after decoupling the coil high frequency resistance, $R_{h f p}^{p}(8)$. Fig. $7 \mathrm{c}$ and $7 \mathrm{~d}$ show the experimentally estimated $R_{h f F E p}^{p}$ vs. the frequency of the injected signal and vs. $H$ respectively. As for $R_{h f p}^{p}, R_{h f F E p}^{p}$ increases with frequency due to the skin effect (see Fig. 7c). It is observed from Fig. $7 \mathrm{~d}$ that $R_{h f F E p}^{p}$ decreases as $H$ increases, meaning that core is slightly affected by the magnetoresistive effect. This was an expected result [13].

Finally, the contribution of the magnet resistance, $R_{h f s}^{p}$, to the overall high frequency resistance can be measured by inserting the magnet in the central column of the core (Fig. $8 \mathrm{a}$ ), once the core and coil contribution to the overall high frequency resistances is decoupled (9). The equivalent circuit in this case is shown in Fig. 8b; the experimentally estimated high frequency resistance is shown in Fig. 8c. It is observed from this figure that $R_{h f s}^{p}$ significantly decreases with $H$, meaning that the magnet is strongly affected by the magnetoresistive effect. 
a)

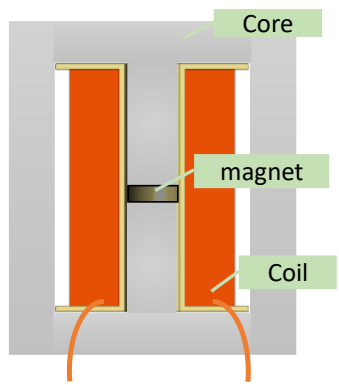

b)

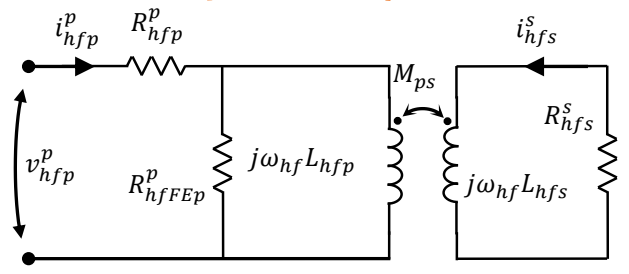

c)
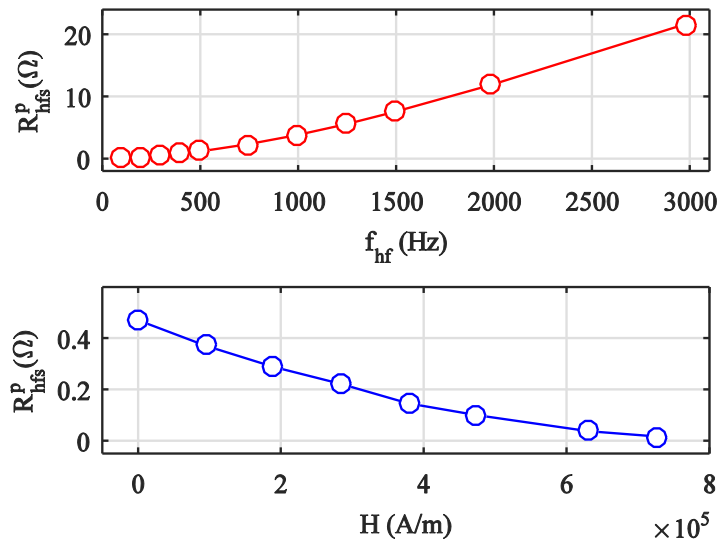

Fig. 8. a) Experimental setup configured for magnet high frequency resistance estimation, b) equivalent high frequency circuit, c) magnet (NdFeB, N42H, see Table III) high frequency resistance for different frequency values, d) $R_{h f p}^{p}$, for different $\mathrm{H}$ values. $f_{h f}=250 \mathrm{~Hz}$ and $I_{h f}=1 \mathrm{~A}$.

\section{EXPERIMENTAL RESULTS}

Experimental results measuring the effect of coating on the magnetoresistive effect using the experimental setup shown in Fig. 3 are presented in this section. Demagnetized and magnetized $\mathrm{NdFeB}$, SmCo and AlNiCo disk shaped magnets have been tested. Two types of coatings, NiCuNi and Epoxy were used. Magnet thickness 5 and $10 \mathrm{~mm}$, were selected all with the same radius (see Fig. 9).

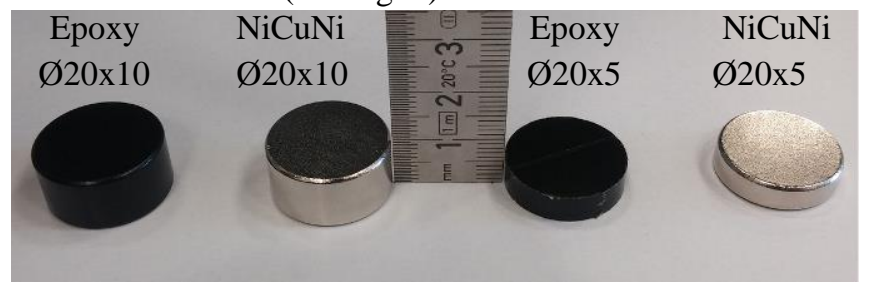

Fig. 9. Magnet shape, size and coating type evaluated for $\mathrm{NdFeB}$, AlNiCo and SmCo.
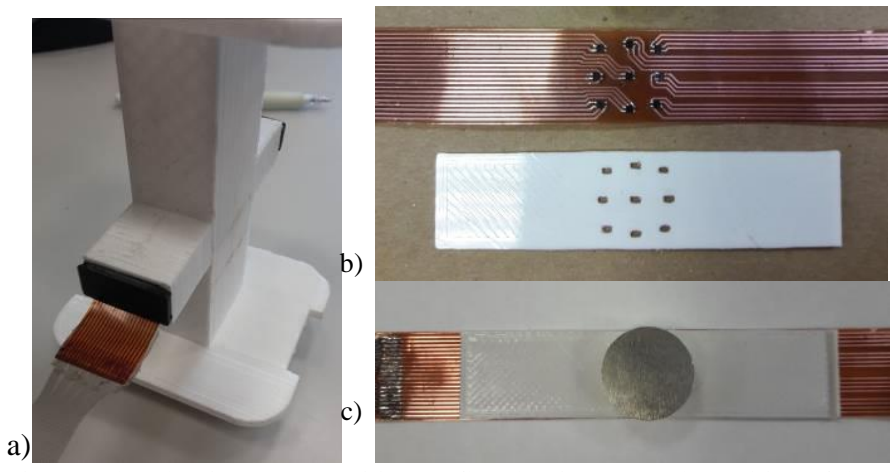

Fig. 10. Flux density sensor position, a) flux density sensor, b) position of the magnet on the sensor array, c).

Epoxy coating presents the same magnetic permeability as air. It was used to keep the same total magnet width, avoiding variations in the equivalent reluctance path of the prototype. In addition, the thickness of the magnet material is also kept constant, avoiding errors due to the differences in volume among samples. Magnets and coating characteristics are summarized in Table III.

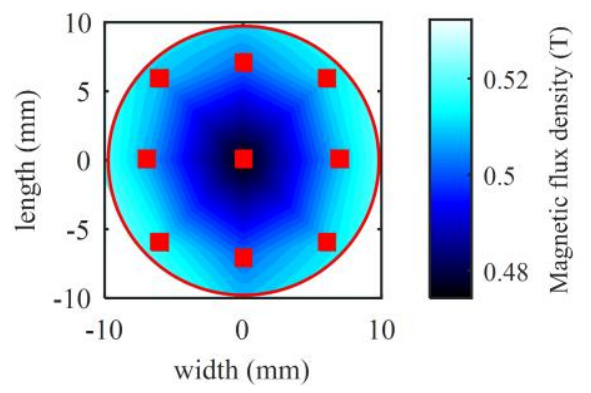

Fig11.Magnetic flux density distribution on magnet surface for a demagnetized $\mathrm{NdFeB}$ magnet when a positive DC field is applied by the coil. Magnet location is indicated by the red circle, sensors location are represented by red squared spots.

TABLE III: MAGNETIC MATERIAL AND COATING THICKNESS

\begin{tabular}{|c|c|c|c|}
\hline Materials & $\begin{array}{c}\text { NdFeB } \\
(\mathrm{N} 42 \mathrm{H})\end{array}$ & $\begin{array}{c}\text { SmCo } \\
(2: 17)\end{array}$ & AlNiCo \\
\hline Ni layer thickness $(\mu \mathrm{m})$ & 3 & 3 & 3 \\
\hline Cu layer thickness $(\mu \mathrm{m})$ & 4 & 4 & 4 \\
\hline $\begin{array}{c}\text { NiCuNi Coating } \\
\text { Thickness }(\mu \mathrm{m})\end{array}$ & 11 & 11 & 11 \\
\hline Epoxy thickness & 6 & 6 & 6 \\
\hline $\begin{array}{c}\text { Magnetic Material } \\
\text { Thickness }(\mathrm{mm})\end{array}$ & 5,10 & 5,10 & 5,10 \\
\hline
\end{tabular}

\section{A. Demagnetized samples}

Figs. 12-14 show the reflected magnet high frequency resistance, $R_{h f s}^{p}$, for the $\mathrm{NdFeB}, \mathrm{SmCo}$ and AlNiCo samples.

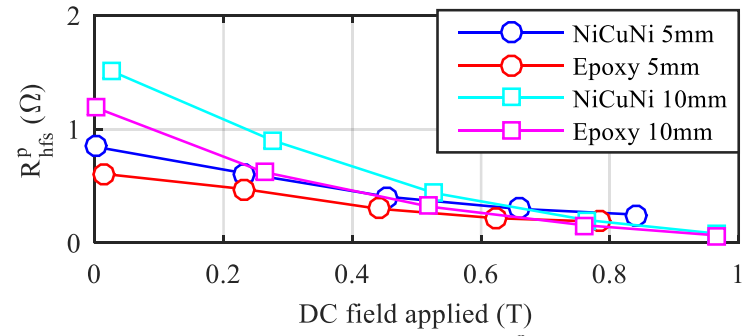

Fig. 12. Reflected high frequency resistance $R_{h f s}^{p}$ of a NdFeB magnet with Epoxy coating, $\mathrm{O}$, and $\mathrm{NiCuNi}$ coating, $\square .20^{\circ} \mathrm{C}, f_{h f}=250 \mathrm{~Hz}$ and $I_{h f}=1 \mathrm{~A}$ 


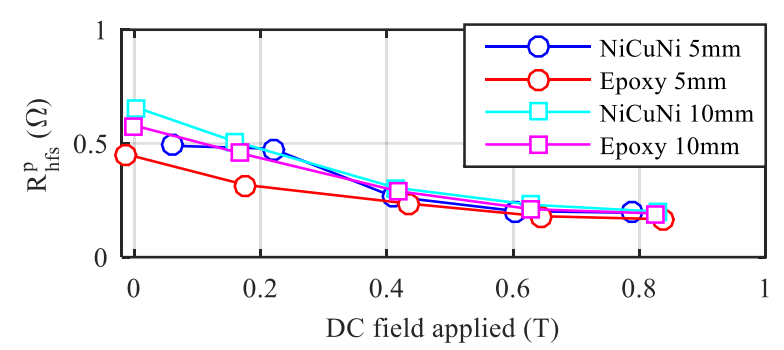

Fig. 13. Reflected high frequency resistance $R_{h f s}^{p}$ of a AlniCo magnet. Same conditions as in Fig. 12

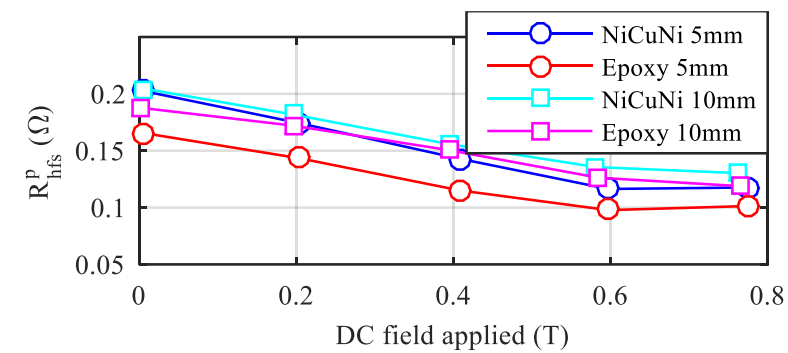

Fig. 14. Reflected high frequency resistance, $R_{h f s}^{p}$ of an SmCo magnet. Same conditions as in Fig. 12.

If magnetic flux density is measured with only one sensor, errors may occur as the magnetization is not uniform along the magnet surface [14]. To avoid this problem, a thin PCB (0.7mm thickness), see Fig. 10, that is equipped with a matrix of $3 \times 3$ Hall sensors was designed to measure the magnet flux density distribution on the magnet surface. The measurement provided by all the nine field sensors will be averaged, and used as a metric of the PM magnetic flux density. Fig. 11 shows an example of a contour plot of the field measured by the Hall sensor array when a DC field of $\approx 0.9 \mathrm{~T}$ is applied to a fully demagnetized magnet by injecting a DC current into the coil (see Fig. 8a).

The demagnetized PMs are inserted in the core as shown in Fig. 8a. The magnet, coil and core temperatures are at room temperature. All magnets were initially fully demagnetized, $B$ being therefore the result of the injected DC current. The high frequency current used for high frequency resistance estimation is superimposed to the DC current. The core and coil high frequency resistances are decoupled from the total estimated high frequency resistance (9), using the data shown in Figs. 6c and 7d.

It can be observed from Figs. 12-14 that the thicker magnets show larger high frequency resistance when no DC field is applied. Most PMs materials show similar values of the estimated high frequency for large values of the DC field $(>0.7 \mathrm{~T})$, independently of the magnet thickness.

The exception is the SmCo magnet, see Fig. 14, magnet with epoxy coating, which shows slightly lower resistance at high DC magnetic fields. The highest variation of the high frequency resistance is observed for $\mathrm{NdFeB}$ magnets. This was expected as this material presents the largest high frequency resistance variations with the magnetization state.

\section{B. Magnetized samples}

$\mathrm{NdFeB}$, AlNiCo and SmCo magnets were evaluated, with and without coating, for five different magnetization levels, i.e. remanent fluxes, of $0,25,50,75$ and $100 \%$ of its nominal value (1.2 T for NdFeB, $0.8 \mathrm{~T}$ for AlNiCo and 0.9 $\mathrm{T}$ for SmCo magnets). Fig. 15-17 and Fig. 18-20 show the experimental results for 5 and $10 \mathrm{~mm}$ thicknesses respectively (see Fig. 9).

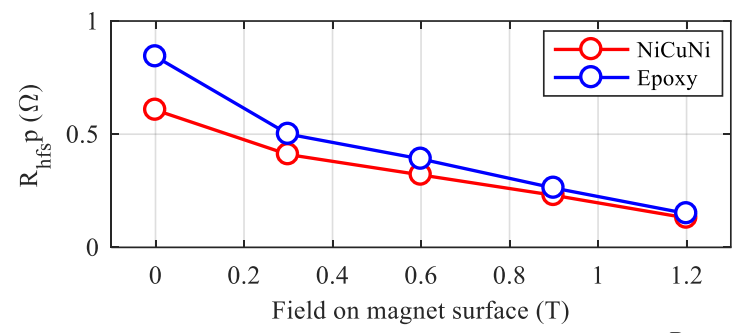

Fig. 15. Reflected PM magnet high frequency resistance $R_{h f s}$ for a $5 \mathrm{~mm}$ thickness magnetized $\mathrm{NdFeB}$ magnet with epoxy, and NiCuNi coating, for different magnetization levels.

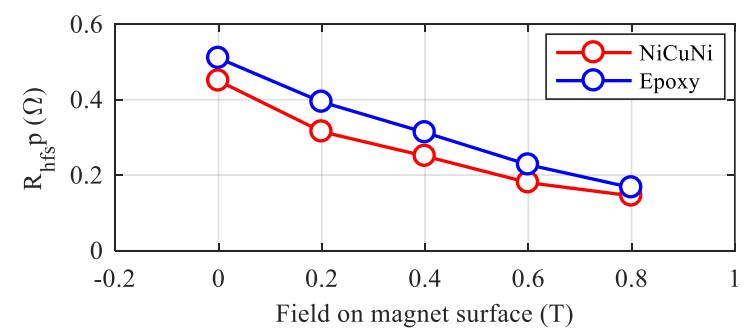

Fig. 16. Same results as in Fig. 15 for an AlNiCo.

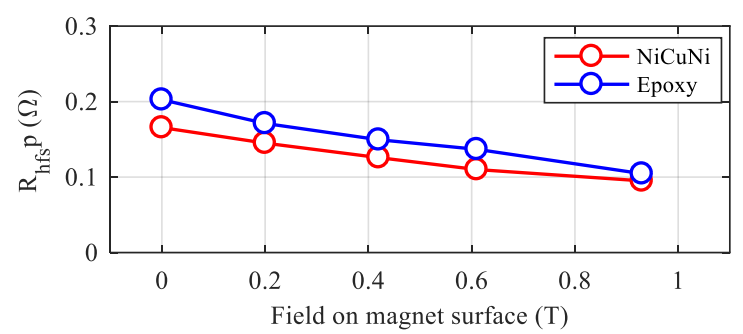

Fig. 17. Same results as in Fig. 15 for a SmCo magnet.

The magnet samples were magnetized using a peak magnetizer shown in Fig 29. The magnetizer parameters are shown in Table IV. Once the samples are magnetized, they are inserted in the core shown in Fig 8a. No DC current is injected in this case, only the high frequency current signal needed for high frequency resistance estimation is being applied. The magnetic field shown in Figs. 15-20 is attributed therefore exclusively to the PM remanent flux. It is observed that in all cases, the higher the magnetization state is, the lower is the reflected high frequency resistance. It is also observed that magnets with $\mathrm{NiCuNi}$ coating show larger high frequency resistance variations, meaning that they are more sensitive to the magnetoresistive effect. The estimated high frequency resistance when the magnetic field is produced externally by the coil (see Figs. 12-14) is different from the estimated high frequency resistance when the flux is produced by the magnet itself. This is mainly due to the differences in the magnetic flux distribution between magnetized magnet and demagnetized magnet within the magnetic circuit (see Figs 11 and 30). 


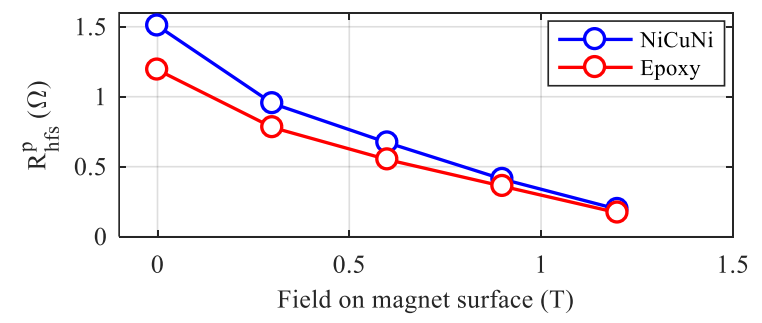

Fig. 18. Same results as in Fig. 15 for a $10 \mathrm{~mm}$ thickness magnetized $\mathrm{NdFeB}$ magnet.

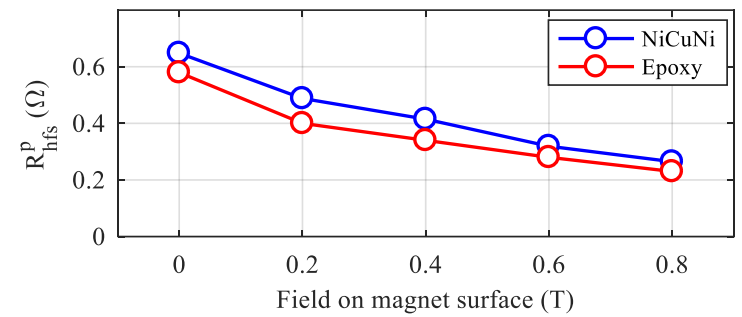

Fig. 19. Same results as in Fig. 16 for a AlNiCo magnet.

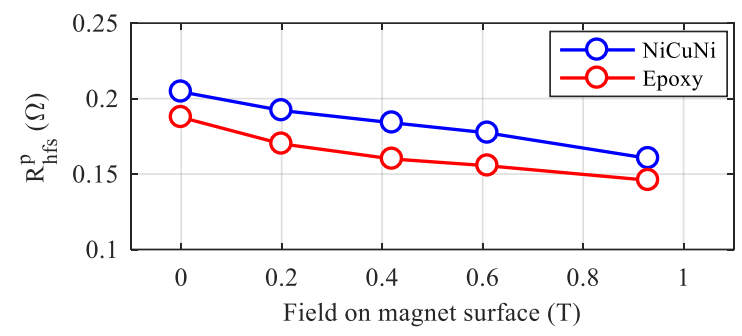

Fig. 20. Same results as in Fig. 17 for a SmCo magnet.

\section{Magnetoresistance in magnetized samples combined with flux-weakening and flux-intensifying current}

$\mathrm{NdFeB}$, AlniCo and SmCo magnets are evaluated, with and without coating, for different magnetization levels, when flux weakening/ intensifying current is applied Fig. 21-23 and Fig. 24-26 show experimental results for 5 and $10 \mathrm{~mm}$ thicknesses (see Fig. 9). Fig. 21 to 23 show the high frequency resistance vs. DC current for two different initial magnetization levels of $\approx 50$ and $100 \%$. Injection of the current for each experiment only lasts XXX, meaning that magnet temperature increase during the experiment can be safely neglected. Coil, core and PM temperatures were monitored during the experiments to confirm this assumption. As in the previous cases, a high frequency signal is superposed to the DC current for high frequency resistance estimation.

For each initial magnetization level, the magnet flux is weakened or intensified by injecting a DC current. It is observed that in all cases the estimated resistance in absence of DC current increases when the permanent magnet flux is weakened and decreases as the magnetic field the magnet surface increases. Different trends are observed by the estimated high frequency resistance for different PM remanences and for magnetized magnets when an external field is used to weaken/intensify its field, no determinant conclusion is reached for this results.

It is concluded from the experimental results that the studied magnet materials coating changes the magnetoresistance effect. The largest high frequency resistance variations are for $\mathrm{NdFeB}$, being slightly smaller for AlNiCo magnets. SmCo magnets show the smallest high frequency resistance variations.

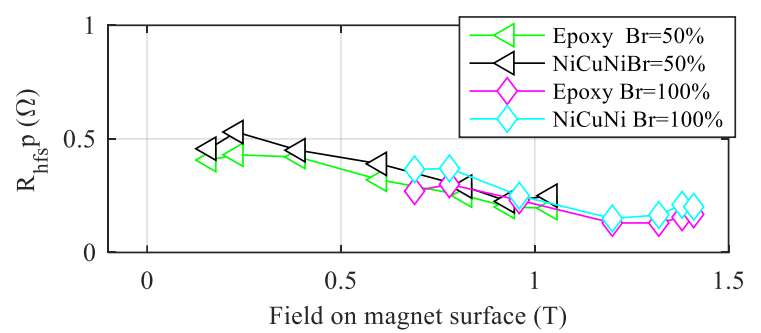

Fig. 21. Reflected PM magnet frequency resistance $R_{h f s}$ for a $5 \mathrm{~mm}$ magnetized $\mathrm{NdFeB}$ magnet with epoxy, and $\mathrm{NiCuNi}$ coating, for different magnetization and flux weakening/ intensifying levels.

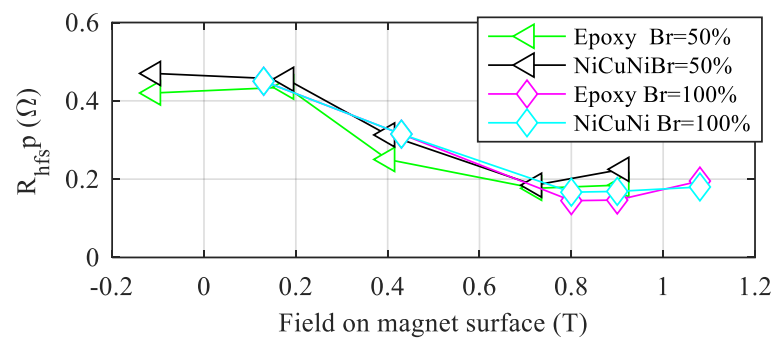

Fig. 22. Same results as in Fig 21 for a magnetized AlNiCo magnet.

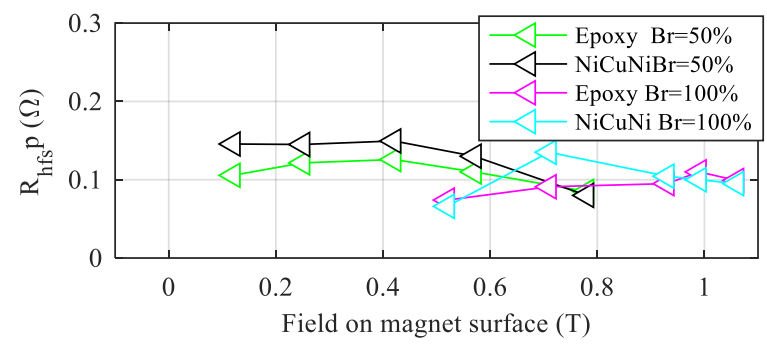

Fig. 23. Same results as in Fig. 21 magnetized SmCo magnet.

\section{A. Frequency of the injected signal}

Fig. 27 shows the magnet reflected high frequency resistance for $\mathrm{NdFeB}$, AlniCo and SmCo magnets, with epoxy and $\mathrm{NiCuNi}$ coatings, and for frequencies ranging from 250 to $1250 \mathrm{~Hz}$. Same as in section IV-A, magnets are fully demagnetized, the field in the magnet surface being therefore due to the injected DC current. The high frequency current used for high frequency resistance estimation is superimposed on top of the DC current. The core and coil high frequency resistances are decoupled. To keep constant coil and core temperatures, the injected DC current has been limited to $15 \mathrm{~A}$, which corresponds to $\approx 0.65 \mathrm{~T}$ on the magnet surface. 


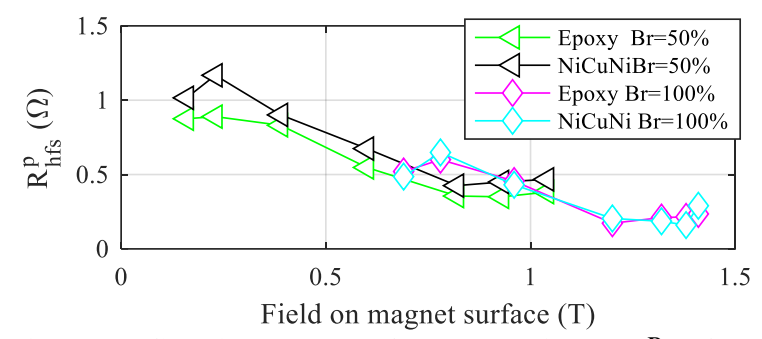

Fig. 24. Reflected PM magnet frequency resistance $R_{h f s}$ for a $10 \mathrm{~mm}$ magnetized $\mathrm{NdFeB}$ magnet with epoxy, and $\mathrm{NiCuNi}$ coating, for different magnetization and flux weakening/ intensifying levels.

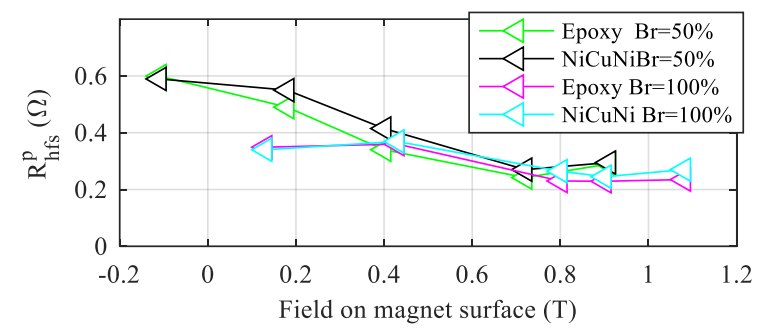

Fig. 25. Reflected PM magnet frequency resistance $R_{h f s}$ for a $10 \mathrm{~mm}$ magnetized AlNiCo magnet with epoxy, and $\mathrm{NiCuNi}$ coating, for different magnetization and flux weakening/ intensifying levels.

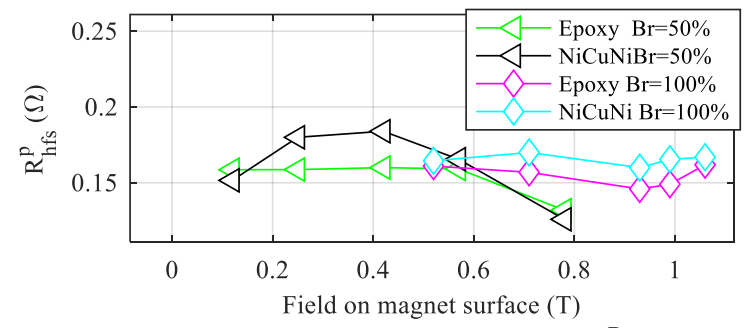

Fig. 26. Reflected PM magnet frequency resistance $R_{h f s}$ for a $10 \mathrm{~mm}$ magnetized SmCo magnet with epoxy, and NiCuNi coating, for different magnetization and flux weakening/ intensifying levels.

It is observed that the frequency of the high frequency signal affects both to the slope (i.e. sensitivity to magnetoresistance effect) and to the reflected magnet high frequency resistance at 0T, i.e. when there is no DC current. Fig. 28 shows the sensitivity (i.e. slopes of the curves in Fig. 27) to magnetoresistive effect of $\mathrm{NdFeB}, \mathrm{SmCo}$ and AlNiCo magnets with $\mathrm{NiCuNi}$ and epoxy coatings at different frequencies. It is observed that both the magnet high frequency resistance at $0 \mathrm{~T}$ (see Fig. 27) and the sensitivity (see Fig. 28) increase as frequency does for all magnets and coatings, the effect being more prominent for $\mathrm{NdFeB}$ magnets. Finally, it is observed that, in all cases, magnets with NiCuNi coating show highest sensitivity to magnetorresistive effect compared to magnets with epoxy coating.
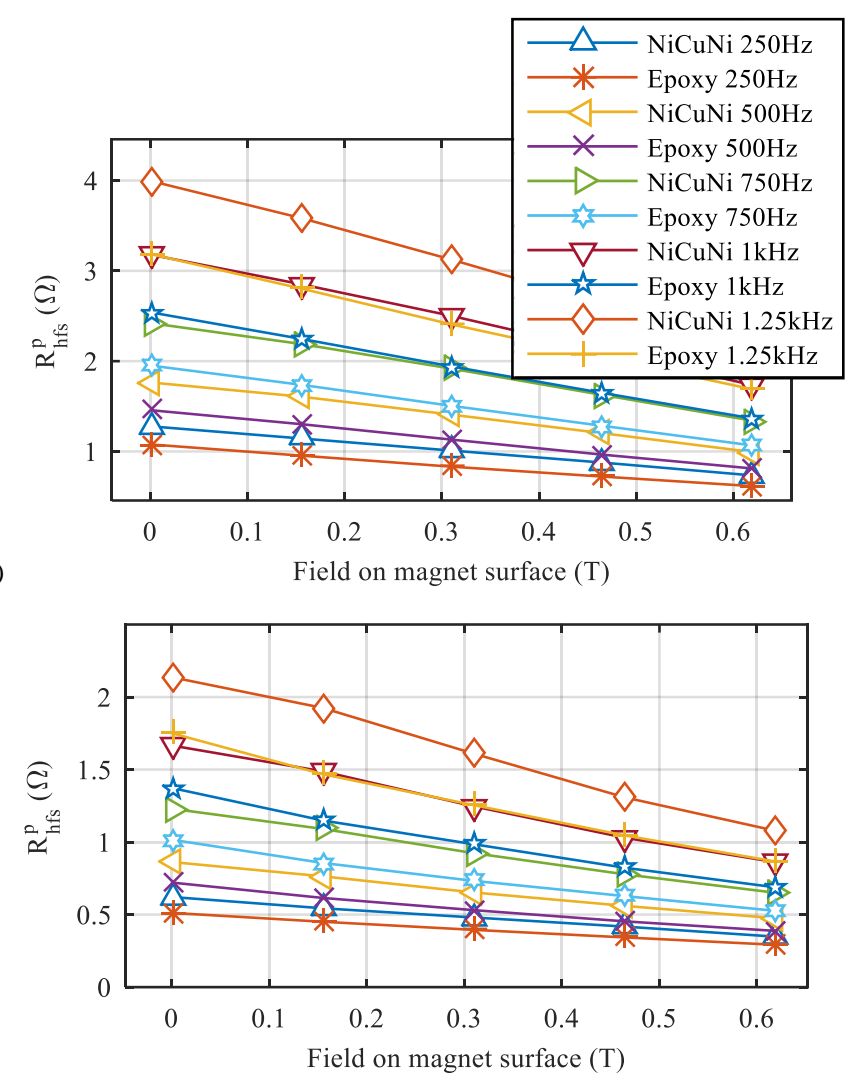

b)

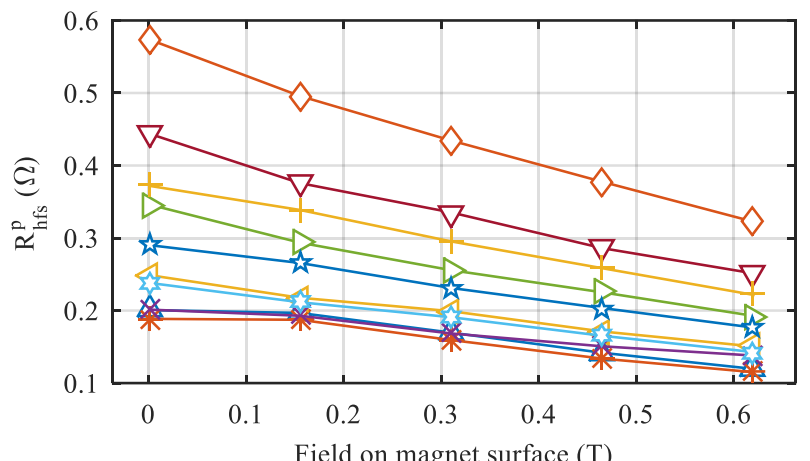

Fig. 27. Reflected high frequency resistance $R_{h f s}^{p}$ of a NdFeB magnet, a), AlNiCo, b), and SmCo, c) with NiCuNi coatings. T $=20^{\circ} \mathrm{C}$ and $I_{h f}=1 \mathrm{~A}$

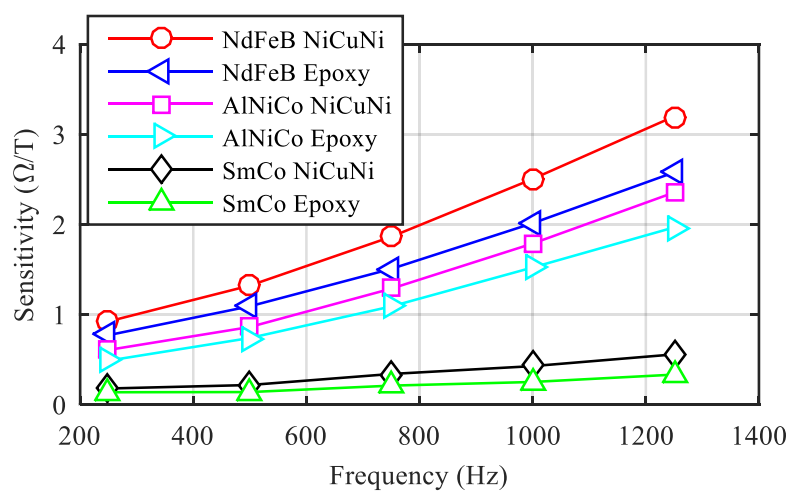

Fig. 28. Sensitivity to magnetoresisitive effect of $\mathrm{NdFeB}, \mathrm{SmCo}$ and AlNiCo magnets with $\mathrm{NiCuNi}$ and epoxy coatings for different frequencies. 


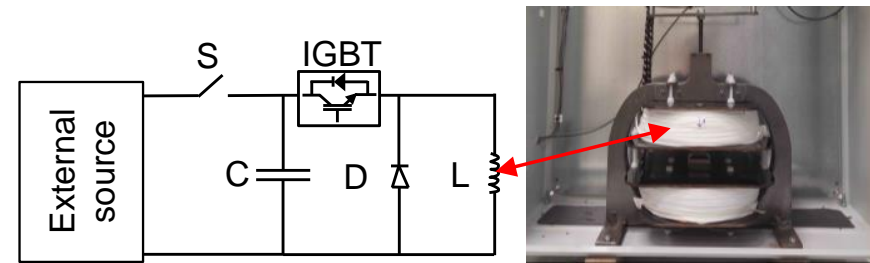

Fig. 29. Schematic representation of the circuit used for PM magnetization and demagnetization.

TABle IV. MAGNETIZATION CiRCUIT PARAMETERS

\begin{tabular}{|l|l|}
\hline External source max. voltage & $750 \mathrm{~V}$ \\
\hline Capacitor "C" & $11750 \mu \mathrm{F}$ \\
\hline Diode "D" & $1000 \mathrm{~V}, 1250 \mathrm{~A}$ \\
\hline IGBT & $1700 \mathrm{~V}, 1400 \mathrm{~A}$ \\
\hline Coil "L" & 1960 turns \\
\hline
\end{tabular}

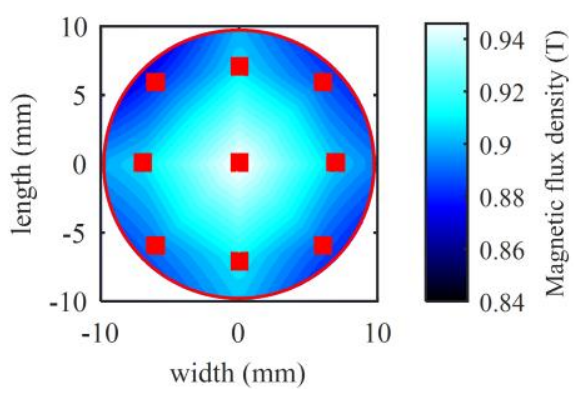

Fig. 30. Magnetic flux density distribution on magnet surface for a fully magnetized NdFeB. Magnet size and position is represented by the red circle, sensors positions represented by red squared spots.

\section{CONCLUSION}

This paper presents an analysis of the effect of PM coating on its magnetoresistance sensitivity, with the goal of estimating the impact of coating on magnetization state estimation methods. The physical principles which explain the effect of coating are the same those of GMR structures. Experimental results using $\mathrm{NdFeB}, \mathrm{SmCo}$ and $\mathrm{AlNiCo}$ magnets with $\mathrm{NiCuNi}$ and epoxy coatings have been presented. It has been demonstrated that $\mathrm{NiCuNi}$ coating improves magnetorresistive effect sensitivity, $\mathrm{NdFeB}$ magnets showing the highest sensitivity increase. Therefore, the use of NiCuNi coating can be advantageous in PMSM machines if MS estimation methods are to be implemented, even if corrosion protection is not required. It has also been shown that the sensitivity to magnetoresistance (NdFeB, SmCo and AlNiCo) can be enhance when coated with $\mathrm{NiCuNi}$ and when the frequency of the injected high frequency signal increases.

\section{REFERENCES}

[1]. T. Kato, N. Limsuwan, C. Y. Yu, K. Akatsu and R. D. Lorenz, "Rare Earth Reduction Using a Novel Variable Magnetomotive Force FluxIntensified IPM Machine," in IEEE Transactions on Industry Applications, vol. 50, no. 3, pp. 1748-1756, May-June 2014.

[2]. H. Yang, Z. Q. Zhu, H. Lin; D. Wu, H. Hua, S. Fang; and Y. Huang, "Novel High-Performance Switched Flux Hybrid Magnet Memory Machines With Reduced Rare-Earth Magnets," in IEEE Transactions on Industry Applications, vol. 52, no. 5, pp. 3901-3915, Sept.-Oct. 2016.

[3]. A. El-Refaie, T. Raminosoa, P. Reddy, S. Galioto, D. Pan, K. Grace, J. Alexander, K. Huh, "Comparison of traction motors that reduce or eliminate rare-earth materials," 2016 IEEE Energy Conversion Congress and Exposition (ECCE), Milwaukee, WI, 2016, pp. 1-8.

[4]. M. Ibrahim, L. Masisi and P. Pillay, "Design of Variable-Flux Permanent-Magnet Machines Using Alnico Magnets," in IEEE Transactions on Industry Applications, vol. 51, no. 6, pp. 4482-4491, Nov.-Dec. 2015. doi: 10.1109/TIA.2015.2461621

[5]. H. Yang, H. Lin, Z. Q. Zhu, D. Wang, S. Fang and Y. Huang, "A Variable-Flux Hybrid-PM Switched-Flux Memory Machine for EV/HEV Applications," in IEEE Transactions on Industry Applications, vol. 52, no. 3, pp. 2203-2214, May-June 2016.

[6]. N. Bianchi and T. M. Jahns "Design, analysis, and control of interior PM synchronous machines," Tutorial Course Notes, IEEE-IAS'04.

[7]. M. Zhu; W. Hu, N. C. Kar, "Torque Ripple Based Interior Permanent Magnet Synchronous Machine Rotor Demagnetization Fault Detection and Current Regulation," in IEEE Transactions on Industry Applications, vol.PP, no.99, pp.1-1 doi: 10.1109/TIA.2016.2634518

[8]. G. Choi; Y. Zhang; T. Jahns, "Experimental verification of rotor demagnetization in a fractional-slot concentrated-winding PM synchronous machine under drive fault conditions," in IEEE Transactions on Industry Applications, vol.PP, no.99, pp.1-1

[9]. N. Limsuwan, T. Kato, K. Akatsu and R. D. Lorenz, "Design and Evaluation of a Variable-Flux Flux-Intensifying Interior PermanentMagnet Machine," in IEEE Transactions on Industry Applications, vol. 50, no. 2, pp. 1015-1024, March-April 2014.

[10]. T. Fukushige, N. Limsuwan, T. Kato, K. Akatsu and R. D. Lorenz, "Efficiency Contours and Loss Minimization Over a Driving Cycle of a Variable Flux-Intensifying Machine," in IEEE Transactions on Industry Applications, vol. 51, no. 4, pp. 2984-2989, July-Aug. 2015.

[11]. X. Zhu, L. Quan, D. Chen, M. Cheng, W. Hua and X. Sun, "Electromagnetic Performance Analysis of a New Stator-PermanentMagnet Doubly Salient Flux Memory Motor Using a Piecewise-Linear Hysteresis Model," in IEEE Transactions on Magnetics, vol. 47, no. 5, pp. 1106-1109, May 2011. doi: 10.1109/TMAG.2010.2072986

[12]. D. Fernandez, D. Reigosa, T. Tanimoto, T. Kato and F. Briz, "Wireless permanent magnet temperature \& field distribution measurement system for IPMSMs," 2015 IEEE Energy Conversion Congress and Exposition (ECCE), Montreal, QC, 2015, pp. 3996-4003.

[13]. D. Diaz Reigosa, D. Fernandez, Z. Q. Zhu and F. Briz, "PMSM Magnetization State Estimation Based on Stator-Reflected PM Resistance Using High-Frequency Signal Injection," in IEEE Transactions on Industry Applications, vol. 51, no. 5, pp. 3800-3810, Sept.-Oct. 2015. doi: 10.1109/TIA.2015.2437975

[14]. D. Fernandez, D. D. Reigosa, J. M. Guerrero, Z. Q. Zhu and F. Briz, "Permanent-Magnet Magnetization State Estimation Using HighFrequency Signal Injection," in IEEE Transactions on Industry Applications, vol. 52, no. 4, pp. 2930-2940, July-Aug. 2016. doi: 10.1109/TIA.2016.2541616

[15]. C. Y. Yu, T. Fukushige, A. Athavale, B. Gagas, K. Akatsu, D. Reigosa, R. D. Lorenz ,"Zero/low speed magnet magnetization state estimation using high frequency injection for a fractional slot variable fluxintensifying interior permanent magnet synchronous machine," IEEEECCE, pp. 2495-2502, Setp. 2014.

[16]. M. F. Hsieh, D. G. Dorrell, C. K. Lin, P. T. Chen and P. Y. P. Wung, "Modeling and Effects of In Situ Magnetization of Isotropic Ferrite Magnet Motors," in IEEE Transactions on Industry Applications, vol. 50, no. 1, pp. 364-374, Jan.-Feb. 2014. doi: 10.1109/TIA.2013.2271472

[17]. Arnoldmagnetics.com, "How To Select The Appropriate Permanent Magnet Material", 2015. [Online]. Available: http://www.arnoldmagn etics.com/en-us/Tech-Library/Technical-Publications [Accessed: 1- Apr -2017].

[18]. Hitachi-metals.co.jp, "Permanent Magnets, Surface Treatments", 2017. [Online]. Available: http://www.hitachimetals.co.jp/e/products/auto/el/p03_21.html [Accessed: 11- Oct -2017].

[19]. S. O. Kasap, "Principles of electronic materials and devices," Third Edition 2006, ISBN 0-07-295791-3.

[20]. Uwe Hartmann, "Magnetic Multilayers and Giant Magnetoresistance," First Edition 2000, ISBN 978-3-642-08487-4. 
[21]. H. Kubota, M. Sato and T. Miyazaki, "Anomalous temperature dependence of giant magnetoresistance in $\mathrm{Ni} / \mathrm{Cu}, \mathrm{Ni}_{95} / \mathrm{Co} 5$ and $\mathrm{Ni}_{95} \mathrm{Fe}_{5} \mathrm{Cu}$ multilayer films" Elsevier, Journal of Magnetism and Magnetic Materials. 167: 12-20, Apr. 1997.

[22]. B. Idzikowski, M. Wolf, A. Handstein, K. Nenkov, F. Stobieski and K.$\mathrm{H}$. Muller, "Inverse giant magnetoresistance in granular $\mathrm{Nd} / \mathrm{sub}$ 2/Fe/sub 14/ B a-Fe," INTERMAG Magnetics Conference, Oct. 1997.

[23]. S. Parkin, Xin Jiang, C. Kaiser, A. Panchula, K. Roche and M. Samant, "Magnetically engineered spintronic sensors and memory," in Proceedings of the IEEE, vol. 91, no. 5, pp. 661-680, May 2003.

[24]. N. Smith, "Micromagnetics of GMR multilayer sensors at high current densities," in IEEE Transactions on Magnetics, vol. 30, no. 6, pp. 38223824, Nov 1994.

[25]. D. Fernandez, D. Reigosa, J. M. Guerrero, Z. Q. Zhu and F. Briz, "Influence of PM coating on PM magnetization state estimation methods based on magnetoresistive effect," 2016 IEEE Energy Conversion Congress and Exposition (ECCE), Milwaukee, WI, 2016, pp. 